\title{
sciendo
}

\section{Test-Retest Reliability of Isokinetic Arm Strength Measurements in Competitive Swimmers}

\author{
by \\ Daniel Collado-Mateo ${ }^{1,2}$, Francisco J. Dominguez-Muñoz', Nuno Batalha ${ }^{3}$, \\ Jose Parraça ${ }^{3}$, Pablo Tomas-Carus ${ }^{3}$, Jose C. Adsuar ${ }^{1}$
}

\begin{abstract}
Swimming motor patterns lead internal rotators to grow stronger than antagonist muscles, what may increase the risk of injury in swimmers. Injury prevention often involves the improvement of external rotators strength, as well as the external rotation/internal rotation ratio. The current research aimed to evaluate the test-retest reliability of shoulder concentric rotation strength in competitive swimmers using an isokinetic dynamometer. The study enrolled 35 competitive swimmers aged between 13 and 19 years. Concentric movements were performed including internal and external rotations of the shoulder joint following the instructions of the standardized protocol. The angular velocity of the test was defined at 60\% $\mathrm{s}$. Outcome measures were peak torque $(\mathrm{Nm})$ and work $(\mathrm{J})$, measured in both, the dominant and non-dominant arms. The external rotation/internal rotation ratio was also calculated. Reliability was excellent for peak torque and work. For the external rotation/internal rotation ratio, the ICC oscillated between 0.744 and 0.860 for the work ratio of the non-dominant arm and the peak torque ratio of the dominant arm, respectively. In general terms, better reliability was observed for peak torque compared with work, for external rotation compared with internal rotation, and for the dominant arm compared with the non-dominant one.
\end{abstract}

Key words: reliability, swimming, injury prevention, sport injuries, peak torque.

\section{Introduction}

Competitive swimming performance is influenced by muscular strength (Garrido et al., 2010; Maszczyk et al. 2012). Shoulder internal rotators have an extremely relevant role in developing propulsive forces responsible for the whole body displacement in freestyle swimming (Holt et al., 2017; Yanai et al., 2000). Upper body force needed to move the swimmer through the water, especially when executing three of the four strokes (freestyle, butterfly, and backstroke), derives primarily from shoulder adduction and internal rotation (Johnson et al., 2003). Thus, shoulder internal rotators and adductors become stronger and hypertrophied unlike their antagonist muscle groups (Weldon Iii and
Richardson, 2001). This hypertrophy can lead to an agonist-antagonist muscle imbalance registered in competitive swimming (Batalha et al., 2013), and might also be associated with an incoming shoulder injury (Weldon Iii and Richardson, 2001). Injury prevention often involves the improvement of external rotators strength. However, the high mobility shoulder joint implies that training programs should also consider the whole joint stabilization. An adequate muscular balance is the joint stabilization basis. In swimmers, the external rotation/internal rotation ratio (ER/IR ratio) is commonly used to evaluate the proportional relationship between agonist and antagonist

1 - Faculty of Sport Sciences, University of Extremadura, Cáceres, Spain.

2 - Instituto de Actividad Fisica y Salud, Universidad Autonoma de Chile, Talca, Chile.

3 - Departamento de Desporto e Saúde, Escola de Ciência e Tecnologia, Universidade de Évora, Évora, Portugal. Research Centre of Sports, Health and Human Development, CIDESD, STRONG Research Community. University of Évora, Évora, Portugal. 
shoulder rotator muscles.

Although the weakness of external rotators is associated with injuries triggered by themselves, the alteration of the agonistantagonist balance seems to be a key factor when developing an injury process (Byram et al., 2010). According to Ellenbecker and Roetert (2003), unilateral ratios characterize the quality of muscular balance. The authors state that the normative shoulder rotator ratio values are between 66 and $76 \%$; thus, values below $66 \%$ are associated with severe imbalances and shoulder joint muscle instability. Therefore, this muscular balance is recommended as a measure for randomized controlled trials in injury preventive programs. In this sense, Swanik et al. (2002) succeeded at improving external rotators strength; however, it also led to greater improvements in internal rotators, which consequently worsened the muscular balance. On the other hand, the training program used by Batalha et al. (2015) induced improvements in the ER/IR ratio $(4.66 \%$ and $7.39 \%$ in the dominant and non-dominant shoulder, respectively), as well as the external rotators strength in swimmers. These two studies used the Biodex dynamometer, considered as the gold standard for assessing muscular strength.

Shoulder internal and external rotators reliability has been previously evaluated in healthy subjects (Edouard et al., 2013; Leggin et al., 1996; Malerba et al., 1993). However, to the best of our knowledge, there is no study assessing the reliability of shoulder's strength measures in swimmers or even athletes practicing other sports disciplines. Since the general population strength levels are likely to be lower than swimmers', reliability statistics may also be different. Therefore, studies assessing reliability in a sample comprised of swimmers are needed.

This study aimed to evaluate the testretest reliability of shoulder concentric rotation strength in competitive swimmers using an isokinetic dynamometer. It was hypothesized that the internal and external rotations, as well as the ER/IR ratio evaluations would be reliable.

\section{Methods}

\section{Participants}

The sample was comprised of 35 swimmers aged between 13 and 19 years (Table 1). Males constituted $71.4 \%$ of the sample, while females $28.6 \%$. The mean body height was 167.91 $\pm 1.38 \mathrm{~cm}$, the body mass index $20.67 \pm 2.41$ and their 2015 FINA point score in the $100 \mathrm{~m}$ freestyle $503.82 \pm 76.49$. The following inclusion criteria were set: a) having qualified to compete in national championships; b) no history of upper limb disorders during the last year; c) a minimum of 8 hours of training per week; d) age between 13 and 20 years old; f) signed written informed consent.

All participants and their legal tutors were informed about the objectives and procedures of the study. All subjects older than 18 gave their written consent to participate in the study. Legal tutors of participants younger than 18 signed the written informed consent form. The Ethics Committee of the seeding Institution (proceeding 09002) approved the study procedures, which followed the Declaration of Helsinki updates.

Measures

The Biodex System 3 Quick-Set isokinetic dynamometer with System 3 software (version 3.40) was used for measuring shoulder rotators strength. The outcome measures were peak torque $(\mathrm{Nm})$ and work $(\mathrm{J})$ in both, the dominant and non-dominant arms. The ER/IR ratio was also calculated for both measures (peak torque and work), and arms (dominant and non-dominant) (Ellenbecker and Roetert, 2003).

\section{Design and procedures}

The same test was performed three times during the same day to evaluate the intra-session test-retest reliability. The first trial was performed for familiarization purposes. Then, the second one was conducted and considered as the "test repetition", and finally the third was the "retest repetition". Only concentric movements were performed including internal and external rotations of the shoulder joint. Test's angular velocity was defined at $60 \% \mathrm{~s}$, and all subjects completed three trials with each shoulder. In studies that aim to obtain shoulder rotator strength, as well as endurance and ER/IR ratios, it is usual to use two speeds, one higher and one lower, being $60 \% \mathrm{~s}$ and $180^{\circ} / \mathrm{s}$ the most commonly used. In order to study PT and work, slow angular velocities are used, since there is an inversely proportional relationship between the angular velocity and the moment of force or torque (Mayer et al., 2001; Varas de la Fuente and 
González Secunza, 2003). On the other hand, in order to access power and fatigue index data, intermediate and / or fast velocities are used (Mayer et al., 2001). However, it should be noted that some authors state that isokinetic evaluations at speeds above $180 \% \mathrm{~s}$ cannot be considered reliable (Mayer et al., 2001), since there are two methodological problems: i) individuals take some time to accelerate the body part to the desired speed; (ii) in concentric contractions at high speeds, there are "torque overshots", and should not be considered in the calculation of the PT. Since we aimed to obtain PT and work, we chose to perform the evaluations at the velocity of $60 \%$ s only.

Since strength levels may widely vary between the dominant and non-dominant arms, both shoulders were evaluated. Each participant was asked to sit in a comfortable position following the standardized protocol instructions (Dvir, 2004). The rest interval between tests equalled two minutes. Raters verbally encouraged the participants during the tests.

Participants were seated and stabilized using velcro straps to avoid compensatory trunk movements. They were positioned with their arms at $90^{\circ}$ of abduction, $90^{\circ}$ of elbow flexion (in the scapular plane), as proposed by other researchers (Julienne et al., 2007). Subjects' position and joint alignment instructions were defined by the isokinetic dynamometer (Wilk, 1991). The procedure was explained to all subjects before starting trials, with emphasis on exerting maximal effort within each one's tolerance. The test began with the arm in full internal rotation by using a range of motion (ROM) of 0 to $90^{\circ}$. ROM halts were used under the manufacturer's recommendations to ensure that the identical ROM was tested bilaterally and during retest conditions. Protocols and assessments were all set for the gravity effect.

\section{Statistical analysis}

Differences between test and retest measures were analyzed using the independent samples $t$ test. Relative and absolute reliability indices were computed under Weir (2005) recommendation. The intraclass correlation coefficient (ICC) was used to evaluate the relative reliability. Specifically, the ICC 3,1 (two-way mixed, single measures) with $95 \%$ confidence intervals for the two repetitions (Shrout and
Fleiss, 1979) was selected. The Standard Error of Measurement (SEM) and the Smallest Real Difference (SRD) were calculated to assess absolute reliability. The SEM was calculated as $\mathrm{SEM}=\mathrm{SD} \cdot \sqrt{1-I C C}$ where SD is the mean SD of both repetitions. This score was subsequently turned into a percentage. The SRD was computed as $1.96 \cdot \mathrm{SEM} \cdot \sqrt{2}$. It was also converted into a percentage since this would enable potential comparisons with further studies.

\section{Results}

Differences between test and retest measurements are reported in Table 2. There was a significant reduction in the peak torque of the external rotation (dominant arm) from test to retest conditions $(p=0.020)$. The retest was not significantly different compared with the test in the remaining variables.

Table 3 summarizes the reliability of isokinetic arm measurements. For the dominant arm, testing of peak torque and rotation work resulted in an ICC higher than 0.95 . The nondominant arm, conversely, was slightly less reliable, with ICC values ranging from 0.917 in internal rotation work $(\mathrm{J})$ to 0.972 in external rotation peak torque $(\mathrm{Nm})$. In general terms, external rotation tests were more reliable than internal rotation ones, while peak torques were slightly more reliable than work.

Regarding the ER/IR ratio, the ICC oscillated between 0.744 for the non-dominant arm work ratio and 0.860 in the dominant arm peak torque ratio. Thus, ICC values of ER/IR ratios were lower than those for the remaining variables.

SEM (\%) and SRD (\%) patterns were close to the one for the ICC values, i.e. a lower percentage for the peak torque compared with work, lower values for external rotation compared with internal rotation, and a lower percentage for the dominant arm compared with the non-dominant one.

\section{Discussion}

This study aimed to evaluate the testretest reliability of shoulder concentric rotation strength in competitive swimmers using a Biodex Isokinetic Dynamometer. The main finding relies upon the excellence of the external and internal rotation testing reliability. 


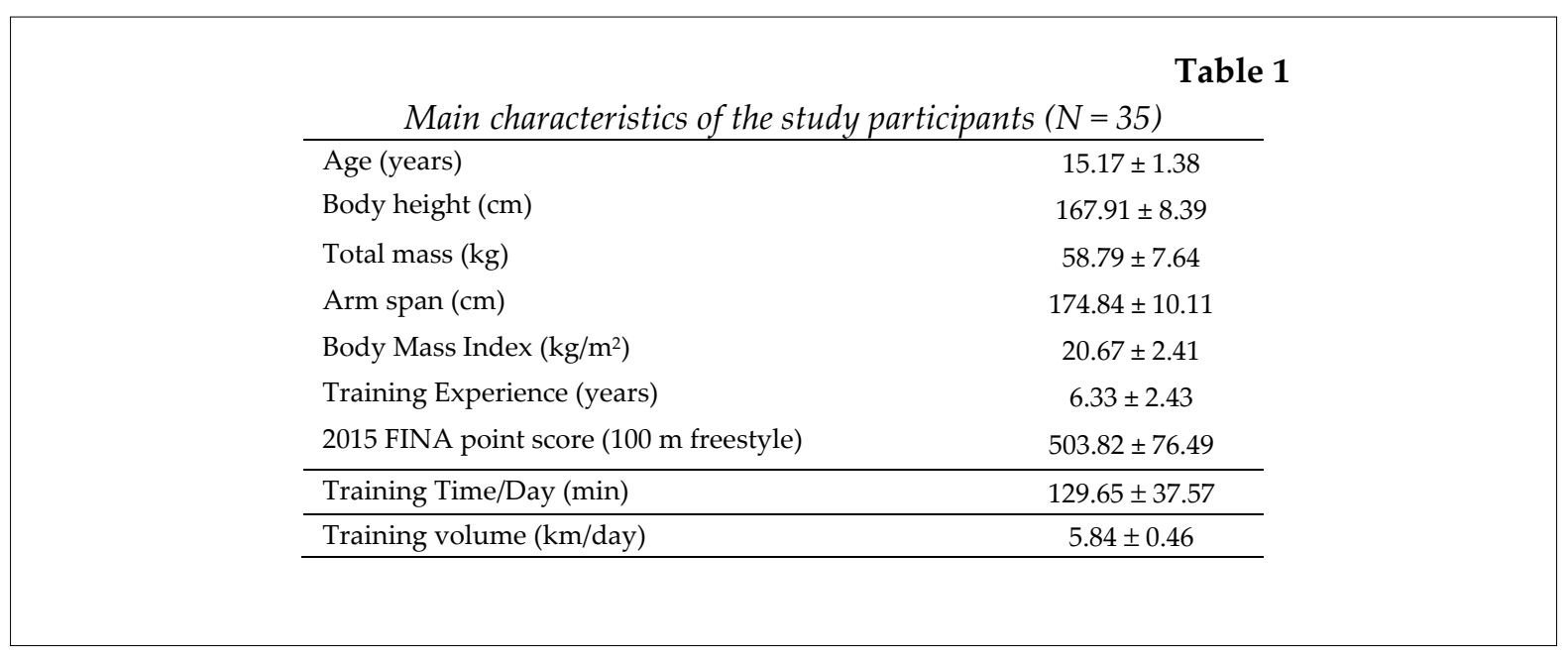

\begin{tabular}{|c|c|c|c|c|}
\hline \multicolumn{5}{|c|}{$\begin{array}{ll}\text { Table } 2\end{array}$} \\
\hline \multirow{2}{*}{ Test Measurement } & \multicolumn{2}{|c|}{ Peak Torque $(\mathrm{Nm})$} & \multicolumn{2}{|c|}{ Work (J) } \\
\hline & Test & Retest & Test & Retest \\
\hline $\begin{array}{l}\text { External Rotation } \\
\text { (Dominant Arm) }\end{array}$ & $24.08 \pm 7.06$ & $23.50 \pm 6.83^{*}$ & $29.42 \pm 8.92$ & $28.98 \pm 8.50$ \\
\hline $\begin{array}{l}\text { Internal Rotation } \\
\text { (Dominant Arm) }\end{array}$ & $28.50 \pm 7.86$ & $28.31 \pm 7.63$ & $36.83 \pm 10.80$ & $37.09 \pm 9.99$ \\
\hline $\begin{array}{l}\text { External Rotation } \\
\text { (No-Dominant Arm) }\end{array}$ & $21.55 \pm 5.91$ & $21.06 \pm 5.52$ & $26.63 \pm 8.13$ & $26.03 \pm 7.27$ \\
\hline $\begin{array}{l}\text { Internal Rotation } \\
\text { (No-Dominant Arm) }\end{array}$ & $28.64 \pm 7.89$ & $27.87 \pm 7.51$ & $36.92 \pm 10.67$ & $36.02 \pm 9.70$ \\
\hline $\begin{array}{l}\text { Ratio External/Internal } \\
\text { (Dominant Arm) }\end{array}$ & $.84 \pm .13$ & $.83 \pm .12$ & $.80 \pm .12$ & $.78 \pm .10$ \\
\hline $\begin{array}{l}\text { Ratio External/Internal } \\
\text { (No-Dominant Arm) }\end{array}$ & $.75 \pm .11$ & $.76 \pm .11$ & $.72 \pm .13$ & $.72 \pm .09$ \\
\hline
\end{tabular}

\begin{tabular}{|c|c|c|c|c|c|c|c|c|c|c|}
\hline & & & & & \multicolumn{6}{|c|}{ Table 3} \\
\hline \multicolumn{11}{|c|}{ Reliability of Isokinetic Arm Measurements $(N=35)$} \\
\hline \multirow[b]{2}{*}{ Test Measurement } & \multicolumn{5}{|c|}{ Peak Torque $(\mathrm{Nm})$} & \multicolumn{5}{|c|}{ Work $(\mathrm{J})$} \\
\hline & $\begin{array}{c}\text { ICC (95\% } \\
\text { CI) }\end{array}$ & $\begin{array}{l}\text { SEM } \\
(\mathrm{Nm}) \\
\end{array}$ & $\begin{array}{c}\text { SEM } \\
(\%)\end{array}$ & $\begin{array}{l}\text { SRD } \\
(\mathrm{Nm})\end{array}$ & $\begin{array}{c}\text { SRD } \\
(\%)\end{array}$ & $\begin{array}{c}\text { ICC (95\% } \\
\text { CI) }\end{array}$ & $\begin{array}{l}\text { SEM } \\
(\mathrm{Nm})\end{array}$ & $\begin{array}{c}\text { SEM } \\
(\%)\end{array}$ & $\begin{array}{l}\text { SRD } \\
(\mathrm{Nm})\end{array}$ & $\begin{array}{l}\text { SRD } \\
(\%)\end{array}$ \\
\hline \multicolumn{11}{|c|}{ Dominant arm } \\
\hline External Rotation Test & $\begin{array}{c}.977 \\
(.955-.988)\end{array}$ & 1.05 & 4.43 & 2.92 & 12.27 & $\begin{array}{c}.967 \\
(.935-.983)\end{array}$ & 1.58 & 5.42 & 4.38 & 15.02 \\
\hline Internal Rotation Test & $\begin{array}{c}.972 \\
(.945-.986)\end{array}$ & 1.29 & 4.56 & 3.59 & 12.65 & $\begin{array}{c}.961 \\
(.924-.980)\end{array}$ & 2.05 & 5.55 & 5.69 & 15.39 \\
\hline $\begin{array}{l}\text { Ratio External/Internal } \\
\text { Rotation Test }\end{array}$ & $\begin{array}{c}.860 \\
(.741-.926)\end{array}$ & .04 & 5.81 & .13 & 16.12 & $\begin{array}{c}.786 \\
(.618-.885)\end{array}$ & .05 & 6.66 & .14 & 18.47 \\
\hline \multicolumn{11}{|c|}{ Non-dominant arm } \\
\hline External Rotation Test & $\begin{array}{c}.972 \\
(.945-.986)\end{array}$ & .95 & 4.49 & 2.65 & 12.44 & $\begin{array}{c}.966 \\
(.934-.983)\end{array}$ & 1.42 & 5.39 & 3.93 & 14.95 \\
\hline Internal Rotation Test & $\begin{array}{c}.938 \\
(.881-.968)\end{array}$ & 1.91 & 6.79 & 5.31 & 18.82 & $\begin{array}{c}.917 \\
(.842-.957)\end{array}$ & 2.93 & 8.04 & 8.13 & 22.30 \\
\hline $\begin{array}{l}\text { Ratio External/Internal } \\
\text { Rotation Test }\end{array}$ & $\begin{array}{c}.782 \\
(.612-.883)\end{array}$ & .05 & 7.25 & .15 & 20.11 & $\begin{array}{c}.744 \\
(.551-.861)\end{array}$ & .05 & 7.92 & .15 & 21.96 \\
\hline ICC: Intraclass Corre & lation Coeffi & $n t ; C$ & $\begin{array}{l}\text { Confid } \\
\text { malles }\end{array}$ & $\begin{array}{l}\text { cee Int } \\
\text { Real D }\end{array}$ & $\begin{array}{l}\text { val; } S \\
\text { erence }\end{array}$ & M: Standa & Errc & fMea & remen & SRD: \\
\hline
\end{tabular}


However, this reliability was reduced from excellent to just good when the ER/IR ratios were analyzed.

According to the results obtained (Table 2 ), the paired-samples $T$ test showed that only the test-retest reduction of the dominant arm external rotation peak torque was significant. Differences between the test and retest were not significant for any other variable. One potential explanation of the considerable test-retest difference could be due to an insufficient rest interval. However, this difference was observed neither in the nondominant arm nor in work. Almost $75 \%$ of the participants achieved a higher peak torque in the test with their dominant arm when compared with the retest, and the remaining $25 \%$ presented lower values. Nevertheless, this tendency was not observed when the non-dominant arm was assessed, since the proportion of participants increasing their torque with their non-dominant arm was close to $50 \%$. This, therefore, may explain why there were no significant changes when assessing their non-dominant arm. Further studies should investigate these intra-session differences between dominant and non-dominant arms using data from electromyography (EMG) or other techniques.

The mean age of the participants was 15.17 years, but results in strength were not very different from those reported by West et al. (2005) at $90 \% \mathrm{~s}$ with a sample comprised by master swimmers aged 43.5 years. As in the current study, ER/IR ratios were slightly higher in that study ( 0.85 for the dominant arm and 0.82 for the non-dominant arm). Therefore, a higher ER/IR ratio was observed in the dominant arm when compared with the non-dominant arm in both studies. This is different from what is observed in other sports such as volleyball, where the ER/IR ratio of the dominant arm is lower than the one observed in the non-dominant arm (Hadzic et al., 2014). Thus, it could be hypothesized that swimming technique, especially the breathing pattern while swimming, could affect the ER/IR ratio. However, this hypothesis should be confirmed in future studies. Although differences between the test and retest were not significant in any of the two ER/IR ratios, the difference between the two arms was reduced from $10.7 \%$ in the test $(0.84$ and 0.75 in the dominant and nondominant arm, respectively), to $8.43 \%$ in the retest
( 0.83 and 0.76 , respectively). This reduction in the difference may indicate that there is a need to explore how the ER/IR ratio change along the repetitions, controlling the effect of fatigue and familiarization. Thus, future studies could investigate reliability assessing arm strength (at least three times) with sufficient rest intervals inbetween.

Results from the current study are relevant due to the imperative role of rotator muscles in swimming performance and injury prevention. Although it is known that the agonistantagonist balance alteration (i.e., ER/IR ratio) is more closely related to the risk of injury than the external rotator muscles weakness (Byram et al., 2010), the relative improvement of that ratio must be higher than the one in the external rotators strength, since the ratio SRD (\%) is higher than the external rotator strength SRD (\%). In this regard, an improvement of at least $16.12 \%$ is needed to achieve a "real change" in the dominant arm for the peak torque ER/IR ratio, while for the work ER/IR ratio the improvement must achieve $18.47 \%$. This improvement needs to be even higher in the non-dominant arm. Therefore, the present study provides relevant information worthwhile to estimate to what extent the progress achieved after an intervention is real or not.

Edouard et al. (2013) evaluated the reliability of shoulder rotator isokinetic strength using the same instrument as the one employed in this study. Participants in that study were healthy adults recruited from a hospital staff. Some differences can be observed when comparing their results with those from the present study. First, although the ICC values for the dominant arm ER/IR ratio were similar, the SEM (\%) and SRD (\%) were about twice the value achieved by swimmers in the current study. Swimmers are often engaged in strength and conditioning programs that may lead to a less muscle imbalance than healthy adults. Furthermore, since swimming is a bilateral sport, swimmers may control better their non-dominant arm strength, which may cause the higher reliability observed in the ratio calculated from the results based on tests performed with that arm. Finally, ICC values of the peak torque were similar to those from this study, but the SEM (\%) and SRD (\%) were higher. Although it is difficult to compare between adults 
and young swimmers, all differences reported in the present study (participants aged $<19$ years) suggest that reliability parameters provided for healthy adults should not be used in a population of young swimmers.

The current study had several limitations. First, the inter-session reliability was not evaluated, thus all absolute and relative statistics reported here may be different when performing both measurements within several days or weeks. Second, the selected test was conducted at $60 \% / \mathrm{s}$, and the reliability may also vary at higher or lower speeds. Finally, the sample size did not allow for the establishment of sub-groups by age and gender. Despite these limitations, this study demonstrates high reliability of the external and internal rotation tests performed with a Biodex Isokinetic Dynamometer in competitive swimmers.

\section{Conclusions}

The reliability of isokinetic arm strength tests performed with a Biodex Isokinetic Dynamometer was excellent in competitive swimmers. In general terms, better reliability was observed for the peak torque compared with work, for external rotation compared with internal rotation, and for the dominant arm compared with the non-dominant one.

\section{Acknowledgements}

The author DCM is supported by a grant from the Spanish Ministry of Education, Culture and Sport (FPU14/01283). The funders had no role in study design, data collection and analysis, decision to publish, or preparation of the manuscript.

\section{References}

Batalha N, Raimundo A, Tomas-Carus P, Paulo J, Simão R, Silva AJ. Does a land-based compensatory strength-training programme influences the rotator cuff balance of young competitive swimmers? Eur J Sport Sci, 2015; 15(8): 764-772

Batalha NM, Raimundo AM, Tomas-Carus P, Barbosa TM, Silva AJ. Shoulder rotator cuff balance, strength, and endurance in young swimmers during a competitive season. J Strength Cond Res, 2013; 27(9): 2562-2568

Byram IR, Bushnell BD, Dugger K, Charron K, Harrell FE, Noonan TJ. Preseason Shoulder Strength Measurements in Professional Baseball Pitchers. Am J Sports Med, 2010; 38(7): 1375-1382

Dvir Z. Isokinetics: muscle testing, interpretation, and clinical applications: Elsevier Health Sciences; 2004

Edouard P, Codine P, Samozino P, Bernard PL, Herisson C, Gremeaux V. Reliability of shoulder rotators isokinetic strength imbalance measured using the Biodex dynamometer. J Sci Med Sport, 2013; 16(2): 162-165

Ellenbecker T, Roetert EP. Age specific isokinetic glenohumeral internal and external rotation strength in elite junior tennis players. J Sci Med Sport, 2003; 6(1): 63-70

Garrido N, Marinho DA, Barbosa TM, Costa AM, Silva AJ, Pérez Turpin JA, Marques MC. Relationships between dry land strength, power variables and short sprint performance in young competitive swimmers. J Hum Sport Exerc, 2010; 5(2): 240-249

Hadzic V, Sattler T, Veselko M, Markovic G, Dervisevic E. Strength Asymmetry of the Shoulders in Elite Volleyball Players. J Athl Train, 2014; 49(3): 338-344

Holt K, Boettcher C, Halaki M, Ginn KA. Humeral torsion and shoulder rotation range of motion parameters in elite swimmers. J Sci Med Sport, 2017; 20(5): 469-474

Johnson JN, Gauvin J, Fredericson M. Swimming biomechanics and injury prevention: new stroke techniques and medical considerations. Phys Sportsmed, 2003; 31(1): 41-46

Julienne R, Gauthier A, Moussay S, Davenne D. Isokinetic and electromyographic study of internal and external rotator muscles of tennis player. Isokinet Exerc Sci, 2007; 15(3): 173-182

Leggin BG, Neuman RM, Iannotti JP, Williams GR, Thompson EC. Intrarater and interrater reliability of 
three isometric dynamometers in assessing shoulder strength. J Shoulder Elbow Surg, 1996; 5(1): 18-24

Malerba JL, Adam ML, Harris BA, Krebs DE. Reliability of dynamic and isometric testing of shoulder external and internal rotators. J Orthop Sports Phys Ther, 1993; 18(4): 543-552

Maszczyk A, Roczniok R, Waśkiewicz Z, Czuba M, Mikołajec K, Zajac A, Stanula A. Application of regression and neural models to predict competitive swimming performance. Percept Mot Skills, 2012; 114(2): 610-26

Mayer F, Horstmann T, Grau S, Handel M, Dickhuth H. Diagnostics with isokinetic devices in shoulder measurements--potentials and limits. Isokinet Exerc Sci, 2001; 9(1): 19-25

Shrout PE, Fleiss JL. Intraclass correlations: uses in assessing rater reliability. Psychol Bull, 1979; 86(2): 420-428

Swanik KA, Swanik CB, Lephart SM, Huxel K. The Effect of Functional Training on the Incidence of Shoulder Pain and Strength in Intercollegiate Swimmers. J Sport Rehabil, 2002; 11(2): 140-154

Varas de la Fuente AB, González Secunza I. Determination of normality by isokinetic evaluation of the musculature of the shoulder joint complex. Rev Iberoam Fisioter Kinesiol, 2003; 6(2): 81-90

Weir JP. Quantifying test-retest reliability using the intraclass correlation coefficient and the SEM. J Strength Cond Res, 2005; 19(1): 231-240

Weldon Iii EJ, Richardson AB. Upper extremity overuse injuries in swimming: A Discussion of Swimmer's Shoulder. Clin Sports Med, 2001; 20(3): 423-438

West D, Sole G, Sullivan SJ. Shoulder external-and internal-rotation isokinetic strength in master's swimmers. J Sport Rehabil, 2005; 14(1): 12-19

Wilk K. Isokinetic Testing-Setup and Positioning. Biodex System II Manual, Applications/Operations, 1991

Yanai T, Hay JG, Miller GF. Shoulder impingement in front-crawl swimming: I. a method to identify impingement. Med Sci Sports Exerc, 2000; 32(1): 21-29

\section{Corresponding author:}

\section{Daniel Collado-Mateo}

Faculty of Sports Sciences

University of Extremadura

Avda. Universidad s/n, 10071 Cáceres, Spain.

Phone: 0034 927257460, Fax: 0034927257461

E-mail: dcolladom@unex.es or danicolladom@gmail.com 